\title{
Beyond Einstein's theory of gravitation?
}

The possibility that the law of gravitation was different early in the history of the Universe seems to be coming into fashion again - and it may just be measurable.

WE all know that Einstein's theory of gravitation is one of the most remarkable and successful theories ever. It is remarkable because there is a sense in which it is the product of pure thought. The starting point is the Equivalence Principle, which goes back to Galileo: there is no way of distinguishing by means of strictly local observations between the circumstances in which an object is accelerated by means of an external gravitational field (as when an apple falls to the Earth) and those in which it is observed from a uniformly accelerated frame of reference.

The identity of the gravitational and inertial masses of an object, often offered as a consequence of the Equivalence Principle, is logically independent. Why, otherwise, would Eötvös in the 1920s have gone to such trouble to measure the gravitational forces on objects of different chemical constitution? And why, otherwise, would there have been such excitement, just a decade ago, in the idea of the "fifth force"?

These were two of the cornerstones of Einstein's theory. He also, of course, knew that any theory of gravitation must merge into Newton's law of gravitation when the masses are small, and that any kinematical implications of the theory must approximate to his own special theory of relativity in appropriate circumstances. His objective was to represent geometrically an arbitrary gravitational field; the outcome was a set of tensor quantities representing the geometry of space-time, with rules for telling where the masses are buried. That is the sense in which the theory is pure thought. It will always be revered as such.

It has also been remarkably successful. The bending of light near the limb of the Sun at the solar eclipse of 1919 was the first validation of Einstein's theory of gravitation. The precession of the perihelion of Mercury came later. The notion that a gravitational field will affect the frequency of a spectral line cannot easily be verified astrophysically, but the prediction has been confirmed by measurements of Mössbauer spectra in the Earth's gravitational field. As it happens, there are few other tests of general relativity than these (but the precession of perihelia now extends to pulsars in elliptical orbit about a companion star).

Whether or not this is a sufficiently rich harvest of validation is a matter of taste, but there is little doubt that Einstein's theory of gravitation has become revered (as it is) because it also accommodates a nat- ural explanation of the expanding Universe. De Sitter's first solution of Einstein's equations expanded spontaneously, but was devoid of matter. Luckily, Friedmann in Moscow showed that there is a whole range of solutions of these difficult equations that will accommodate matter (provided that it is distributed homogenously and isotropically). These are the solutions on which all present models of the Universe are based.

To be sure, there are a few clouds on the horizon, but they seem hardly "bigger than a man's hand". One difficulty is that general relativity allows black holes to exist, with all the disbelief that that requires of the rest of us. The most serious difficulty is that, after more than a quarter of a century, there is still no way of reconciling Einstein's theory of gravitation, his general theory of relativity, with quantum physics. A generation of talented people, not so much an army as a company, has beaten its head against that problem without much success. Some have begun wondering whether there is some other way, perhaps a theory of gravitation that is easily reconcilable with quantum mechanics or, otherwise, does not allow the dense concentrations of matter that make quantum mechanics necessary.

Now there is fresh hope that they may have nature on their side, and even that their optimism may be verifiable. Nostalgia enters at this point. A quarter of a century ago, there was the Brans-Dicke alternative to Einstein's theory. Following the same intellectual construction as Einstein's, it included a scalar as well as a tensor field in its description of space-time. It was eventually undermined by the recognition that the scalar field would now be much more conspicuous than observations of the real world will allow. Unfortunately for Brans and Dicke, their theory was decently put to rest before Alan Guth invented the notion that, in the very earliest moments of the evolution of the Universe, a few microseconds after the Big Bang, the condensation of radiation into matter caused a huge inflation of the physical size of the Universe (by a factor of $10^{50}$ or thereabouts).

Two years ago, Thibault Damour and Kenneth Nortvedt from the Institut des Hautes Etudes at Bures sur Yvette (but with separate affiliations to the Observatoire de Paris and the University of Montana respectively) looked again at scalar-tensor theories of gravitation and concluded that inflation would upset earlier conclusions (Phys. Rev. Lett. 70, 2217-2219; 1993). In particular, they showed that, in an evolving Universe, the scalar part of a scalar-tensor field would rapidly decay as a consequence of its inherent structure. No traces of the scalar field would be left at the present time. But a bout of inflation could cure that, leaving a barely measurable curvature of space-time as a proof that the space-time metric had once had a scalar component.

Benjamin Lange, from the Department of Physics at Stanford University, believes he has a way of measuring this curvature. $\mathrm{He}$ wants to put into an orbit about the Earth a gyroscope whose stability would far outdo the (mostly) gedanken experiments of the late William Fairbank of the same university. (Phys. Rev. Lett. 74, 1904-1907; 1995). It is an astonishing device: a $5-\mathrm{cm}$ sphere of doped silicon spinning in empty space without support.

Naturally, even in a high orbit, a spinning sphere meant to act as a gyroscope must be protected from drag; why not enclose it is a spherical container, or satellite, which can also conveniently carry the equipment meant to read out data from the spinning sphere? The obvious objection that the casing (exposed to drag) and the sphere (free from it) would then collide is met by the simple device of measuring where the sphere is and arranging that the satellite and its equipment keep their designed distance.

That, of course, is almost child's stuff now. The professional cleverness in Lange's scheme is to use a set of Helmholtz coils to spin up the gyroscope and to collect data from the system. In a helpful letter, Lange explains that he hopes to measure the orientation of the axis of the spinning sphere to as little as 0.04 microseconds of arc by means of collimating instruments looking at optically flat surfaces at the two poles of the sphere. To cancel out the proper motion of the star on which the telescope is aligned, it would be better to have two counter-rotating satellites in orbit at the same time.

And the pay-off? If there were ever a scalar field, its remnant might just be measurable. Lange argues that his satellite gyroscope is more likely than any other device, by an order of magnitude, to tell whether gravitation now is different from what it used to be in the early Universe. And that would be a prize worth having.

John Maddox 